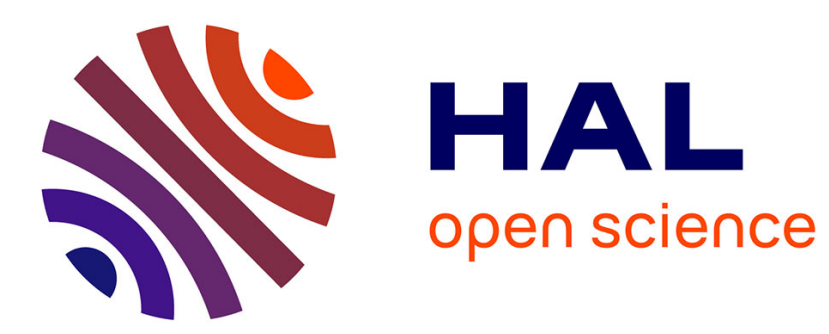

\title{
Pseudo multivariate morphological operators based on alpha-trimmed lexicographical extrema
}

\author{
Erchan Aptoula, Sébastien Lefèvre
}

\section{To cite this version:}

Erchan Aptoula, Sébastien Lefèvre. Pseudo multivariate morphological operators based on alphatrimmed lexicographical extrema. 5th International Symposium on Image and Signal Processing and Analysis (ISPA), 2007, Turkey. pp.367-372, 10.1109/ISPA.2007.4383721 . hal-00516074

\section{HAL Id: hal-00516074 \\ https://hal.science/hal-00516074}

Submitted on 8 Sep 2010

HAL is a multi-disciplinary open access archive for the deposit and dissemination of scientific research documents, whether they are published or not. The documents may come from teaching and research institutions in France or abroad, or from public or private research centers.
L'archive ouverte pluridisciplinaire $\mathbf{H A L}$, est destinée au dépôt et à la diffusion de documents scientifiques de niveau recherche, publiés ou non, émanant des établissements d'enseignement et de recherche français ou étrangers, des laboratoires publics ou privés. 


\title{
Pseudo Multivariate Morphological Operators based on $\alpha$-trimmed Lexicographical Extrema
}

\author{
Erchan Aptoula and Sébastien Lefèvre \\ UMR-7005-CNRS-LSIIT Louis Pasteur University, \\ Pôle API, Bvd Brant, PO Box 10413, 67412 Illkirch Cedex, France, \\ \{aptoula,lefevre\}@lsiit.u-strasbg.fr
}

\begin{abstract}
The extension of mathematical morphology to color and more generally to multivariate image data is still an open problem. The definition of multivariate morphological operators requires the introduction of a complete lattice structure on the image data, hence vectorial extrema computation methods are necessary. In this paper, we propose a lexicographical approach with this end, based on the principle of $\alpha$-trimming, that leads to flexible, but nevertheless pseudo-morphological operators, in the sense that there is no underlying binary ordering relation among the vectors. Moreover a possible solution to this problem is presented as well as a way of automatically computing the parameter $\alpha$ based on statistical measures. The results of a series of color noise reduction experiments are also included, illustrating the superior performance of the proposed approach against uncorrelated Gaussian noise, with respect to stateof-the-art vector ordering schemes.
\end{abstract}

\section{Introduction}

The extension of mathematical morphology to multivariate image data, and specifically to color images is still an open problem. Since the early 1990s, when the underlying theory was formalized $[4,11,12]$, and the complete lattice theory was accepted as its appropriate algebraic basis, several approaches were proposed to this end. The requirement of this extension, consisting of a vector ordering that would provide the vectorial extrema necessary for inducing a complete lattice structure on the image data, has led to various multivariate morphological frameworks, of which none has yet been widely accepted. Besides the inherent difficulty of ordering vectorial data, the nature of color has also proven to be an additional obstacle in this regard. A comprehensive survey on the different approaches can be found in [2].

One of the most popular vector ordering mechanisms used in this area is the standard lexicographical approach, which however suffers from the extreme prioritization of the first dimension, hence ignoring largely the rest of the vector components. In this paper, we address this problem, and propose a modified version of lexicographical ordering based on the principle of $\alpha$-trimming, borrowed from the area of noise reduction, where it has been long employed against impulsive noise in the form of the $\alpha$-trimmed mean filter and its variants [9]. Although the resulting $\alpha$ trimmed lexicographical extrema computation scheme provides a more flexible solution with interesting filtering capabilities, we show that the morphological operators thus obtained are theoretically unsound and present a workaround for this problem. We further discuss the choice of the $\alpha$ argument and propose a computation method in this end based on the standard deviation of image channels.

Additionally, the results of a series of tests are provided, where the proposed approach is compared with state-of-theart vector orderings, in the context of colour noise reduction. Its superior performance against Gaussian noise illustrates its practical interest, in spite of its theoretical deficiencies.

The rest of the paper is organized as follows. Section 2 introduces briefly the theoretical aspects of extending morphological operators to multivariate images and some of the related methodologies. In section 3, the proposed approach is presented and its theoretical properties are elaborated. Then, in section 4, we discuss the results obtained from the comparative tests. Finally section 5 is devoted to concluding remarks.

\section{Multivariate Mathematical Morphology}

In this section we review briefly the main issues concerning the extension of mathematical morphology to multivariate images. For an in-depth study of this topic the reader can refer to the fundamental references $[4,12]$.

\subsection{Definitions}

Among the different methodologies that appeared with the end of extending binary morphological operators to greyscale images, the one based on complete lattices received the widest acceptance and is now considered as the right mathematical framework for morphology [11]. According to this approach, given an image $f: \mathcal{E} \rightarrow \mathcal{T}$ with 
$\mathcal{E}$ an arbitrary non empty set, a complete lattice structure is imposed on the grey-level range $\mathcal{T}$. In other words, $\mathcal{T}$ must be a non empty set equipped with a partial ordering such that every non empty subset $\mathcal{P} \subseteq \mathcal{T}$ has a greatest lower bound $\bigwedge \mathcal{P}$ (infimum) and a least upper bound $\bigvee \mathcal{P}$ (supremum). Consequently, while the scalar order is sufficient for verifying this condition in the case of greyscale images, where usually $\mathcal{T}=\overline{\mathbb{Z}}$ or $\mathcal{T}=\overline{\mathbb{R}}$, it becomes a much more challenging task with multivariate images, where usually $\mathcal{T}=\overline{\mathbb{Z}}^{n}$ or $\mathcal{T}=\overline{\mathbb{R}}^{n}$ with $n>1$, since there is no natural ordering relation for multivariate data.

Indeed, given such a vectorial ranking scheme " $<$ ", the vectorial versions of the two fundamental morphological operators, erosion $\left(\varepsilon_{b}\right)$ and dilation $\left(\boldsymbol{\delta}_{b}\right)$ of a multivalued image $\mathbf{f}$ by a flat structuring element (SE) $b$, can be immediately derived by means of the vectorial extrema operators $\sup _{v}$ and $\inf _{v}$ based on the given ordering:

$$
\begin{aligned}
& \varepsilon_{b}(\mathbf{f})(\mathbf{x})=\inf _{v}\{\mathbf{f}(\mathbf{x}+\mathbf{s})\} \\
& \boldsymbol{\delta}_{b}(\mathbf{f})(\mathbf{x})=\sup _{\mathbf{s} \in b}\{\mathbf{f}(\mathbf{x}-\mathbf{s})\}
\end{aligned}
$$

Therefore, the main obstacle preventing the extension of morphological operators to multivalued images, consists in defining a vector ordering relation that will induce a complete lattice structure on the image data.

\subsection{Vector orderings}

Vector ordering methodologies have been studied relatively thoroughly in the literature [3], and numerous solutions have been proposed in this end. Some of the most popular of which include the marginal or M-ordering, which is a partial ordering realized in a componentwise fashion, hence neither exploiting the inter-channel relations nor preserving the original vectors. This last property renders marginal ordering inadequate for color images as it introduces false colors. Reduced or R-orderings on the other hand, first reduce vectors into scalar values, and then order them according to the natural scalar order. However, unless the reduction transformation is injective, this approach results in pre-orderings that do not lead necessarily to unique extrema.

Conditional or C-orderings, order vectors by means of some of their marginal components, selected sequentially according to different conditions, with the lexicographical ordering " $<_{L}$ " being a widely known example of this group:

$$
\begin{aligned}
& \forall \mathbf{v}, \mathbf{v}^{\prime} \in \mathbb{R}^{n}, \quad \mathbf{v}<_{L} \mathbf{v}^{\prime} \Leftrightarrow \\
& \exists i \in\{1, \ldots, n\}, \quad\left(\forall j<i, v_{j}=v_{j}^{\prime}\right) \wedge\left(v_{i}<v_{i}^{\prime}\right)
\end{aligned}
$$

From a theoretical point of view, lexicographical ordering is a total ordering, thus preserving the input vectors and producing unique extrema. That is why most efforts in the area of multivariate morphology, and color in particular, are based upon it [1, 7].

However, despite its theoretical advantages, most usually in practice the vast majority of lexicographical comparisons are determined by means of the first component
[5]. The remaining dimensions hardly participate in this process, hence leading to an inefficient exploitation of the inter-channel relations. In order to remedy this problem, a first attempt was made by Ortiz et al. [8], that proposed the $\alpha$-lexicographical ordering:

$$
\begin{aligned}
& \forall \mathbf{v}, \mathbf{v}^{\prime} \in \mathbb{R}^{n}, \mathbf{v}<\mathbf{v}^{\prime} \Leftrightarrow \\
& \left\{\begin{array}{l}
v_{1}+\alpha<v_{1}^{\prime}, \text { or } \\
v_{1}+\alpha \geq v_{1}^{\prime} \text { and }\left[v_{2}, \ldots, v_{n}\right]^{T}<_{L}\left[v_{2}^{\prime}, \ldots, v_{n}^{\prime}\right]^{T}
\end{array}\right.
\end{aligned}
$$

where $\alpha \in \mathbb{R}$, and for high values of $\alpha$, comparisons reach more frequently the second dimension. Nevertheless, expression (4) is not transitive, hence does not represent an ordering from an algebraic point of view. A theoretically more sound methodology was introduced by Angulo [1], the $\alpha$-modulus lexicographical ordering:

$$
\begin{aligned}
& \forall \mathbf{v}, \mathbf{v}^{\prime} \in \mathbb{R}^{n}, \mathbf{v}<\mathbf{v}^{\prime} \Leftrightarrow \\
& {\left[\left\lceil v_{1} / \alpha\right\rceil, v_{2}, \ldots, v_{n}\right]^{T}<_{L}\left[\left\lceil v_{1}^{\prime} / \alpha\right\rceil, v_{2}^{\prime}, \ldots, v_{n}^{\prime}\right]^{T}}
\end{aligned}
$$

which by means of a division by a constant $\alpha$ followed by a rounding off reduces the dynamic margin of the first component (considered as integer values in $[0,255]$ ), thus allowing a greater number of comparisons to reach the second.

\section{3. $\alpha$-trimmed lexicographical extrema}

Here, we propose an alternative measure, that can be employed in order to obtain a less "asymmetric" lexicographical ordering, based on the $\alpha$-trimming principle used in filters such as the $\alpha$-trimmed mean filter $(\alpha \mathrm{MF})$ [9]. Given a vector $\mathbf{v} \in \mathbb{R}^{n}$, containing the sorted scalar pixels under the filtering window, the underlying idea of $\alpha$-trimming consists in computing their mean by ignoring the $2 \alpha$ extreme:

$$
\alpha \operatorname{MF}(\mathbf{v})=\frac{1}{n-2 \alpha} \sum_{i=\alpha+1}^{n-\alpha} v_{i}
$$

where $\alpha \in[0, n / 2]$, and $n$ is odd.

\subsection{Proposed approach}

Similarly, in the case of multidimensional vectors we can apply the same principle to each dimension in an iterative fashion. Specifically, in the case of the maximum, starting from the first dimension, we can sort all vectors according to this dimension, and then keep the $\alpha$ greatest. Consequently, at each step the initial set of vectors will get smaller. Against the eventual situation, where more than one vectors remain at the end of this procedure, we choose to use the last dimension for tie-breaking purposes. A more formal description for computing the maximum based on this procedure, is given in table 1 .

As to the minimum, it can be obtained in a likewise fashion by simply sorting in a decreasing order. An illustration of this approach on a three dimensional space $D 1 \times D 2 \times D 3$ 

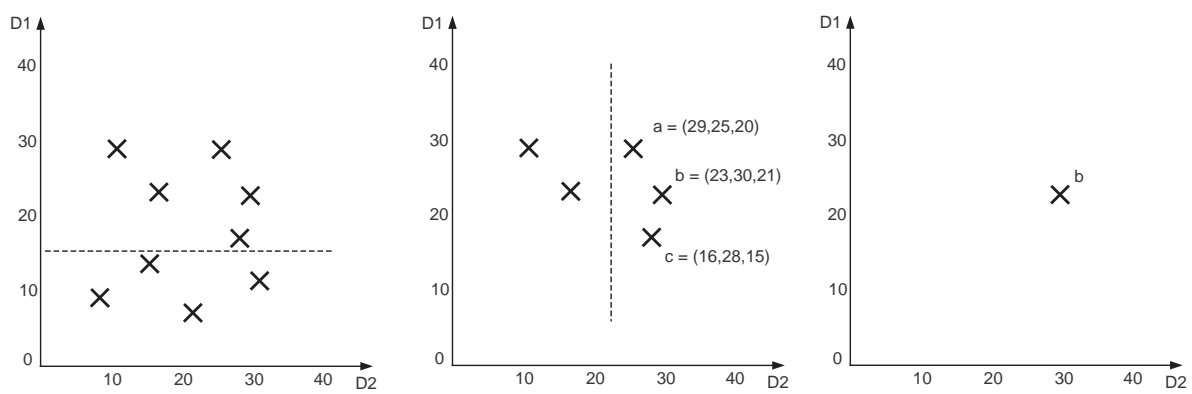

Figure 1. A set of vectors in a three dimensional space $D 1 \times D 2 \times D 3$, and the three iterations of the $\alpha$-trimmed lexicographical maximum computation with $\alpha=0.5$ (left to right). According to the proposed approach, the maximum will be the greatest, with respect to the third dimension, of the remaining three vectors.

\section{Table 1. The $\alpha$-trimmed lexicographical maxi- mum computation algorithm}

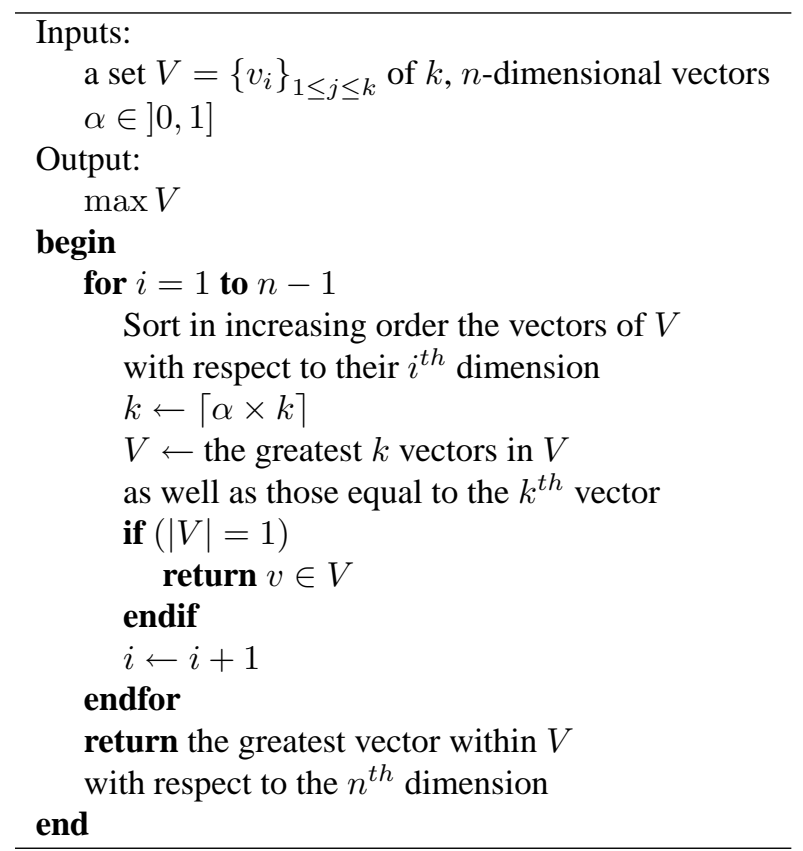

is given in figure 1 . Of course the value of $\alpha$ greatly influences the choice of extrema. As a matter of fact, with an $\alpha$ approaching zero, from each dimension $i$, only the extreme (i.e. maximum or minimum) vector is kept along with those equal to it with respect to dimension $i$. In other words the procedure becomes identical to the standard lexicographical ordering. On the other hand, when $\alpha$ approaches one, priority is shifted gradually to the last dimension. Additionally, more complicated priority relations among the available channels can be established by means of different $\alpha$ values for each vector dimension.

As far as its computational complexity is concerned, as- suming an optimal sorting procedure is used, in the worst scenario, where all $n$ dimensions would need to be sorted, with $k$ vectors the complexity would be in the order of $O(n \times k \times \log k)$. Whereas for the same scenario, the complexity of computing an extremum by means of a lexicographical ordering would be $O(n \times k)$. Besides this additional computational cost, the flexibility of this approach is coupled with an even more important drawback.

Specifically, according to section 2.1, in order to obtain theoretically correct morphological operators, at least a partial ordering needs to be induced on the image data. The proposed extremum computation method however does not have an underlying binary relation, let alone an ordering. All the same, since unique extrema can be computed, the erosion and dilation operators as well as those derived from them can still be defined, however none of the standard morphological properties can be guaranteed (e. g. idempotence, increasingness, etc), hence they are called "pseudomorphological operators". A further example of this type of an approach consists of the extrema obtained by cumulative distances, where the minimum is defined as the median vector and the maximum as the most distant. Given $V=\left\{v_{i}\right\}_{1 \leq j \leq k}$ a set of vectors:

$$
\begin{aligned}
& \max V=\arg \max _{i}\left\{\sum_{j \neq i} d\left(v_{i}, v_{j}\right)\right\} \\
& \min V=\arg \min _{i}\left\{\sum_{j \neq i} d\left(v_{i}, v_{j}\right)\right\}
\end{aligned}
$$

where $d(\cdot, \cdot)$ denotes a distance.

Although a serious handicap, the lack of an underlying ordering relation does not hinder the practical use of pseudo-morphological operators based on these extrema computation schemes.

For instance Plaza et al. in [10] employ successfully extended morphological profiles calculated using operators based on equations (7) and (8) for the classification of mul- 


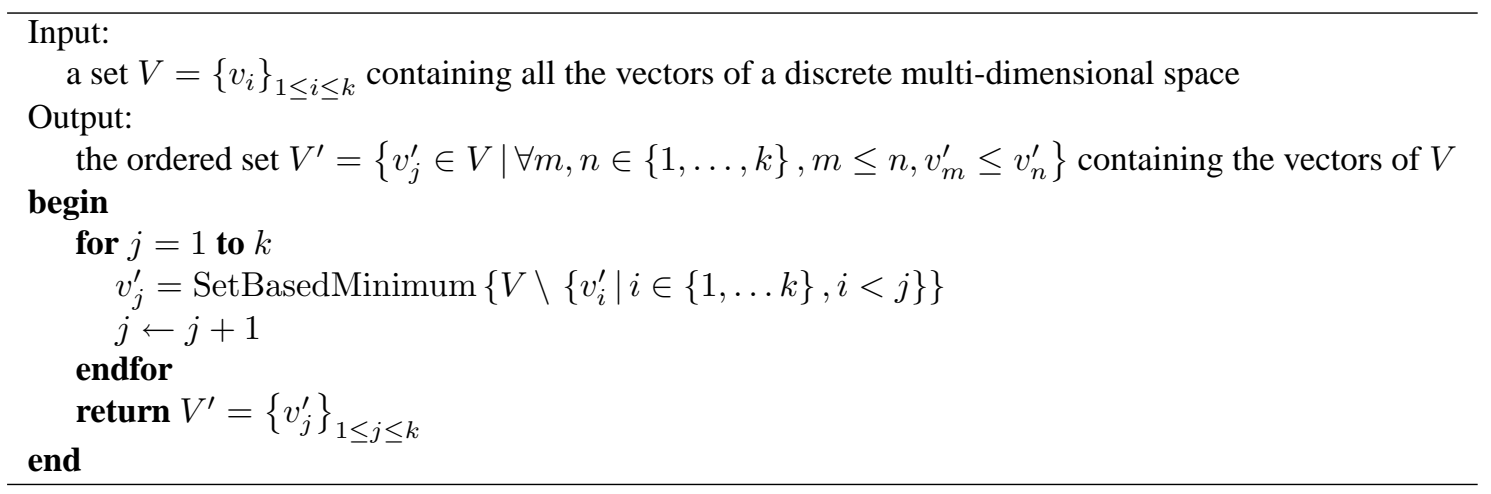

Table 2. The ordering of a multi-dimensional space using a set-based minimum computation method (SetBasedMinimum)

tispectral remote sensing images. In fact, thanks to the "setbased" computation of the extrema, conversely to binary relations, these approaches can be presumed to better exploit the distribution of the vectors within the multi-dimensional space, thus often exhibiting interesting behaviors (section 4).

\subsection{From set-based to binary relations}

Furthermore, in situations where binary orderings are a necessity, besides using one of the many available alternatives one can also employ the set-based approaches in order to derive a binary ordering. Specifically, given a discrete multi-dimensional space, one can employ the set-based extremum computation method at hand, in order to calculate the maximum or minimum of this space, and then repeat for the remaining points, up until the entire space is ordered. The formal procedure is described in table 2.

Consequently, given then any two vectors within this space their order is known. Moreover, since $\alpha$-trimmed lexicographical extrema are unique, this type of an approach would lead to a total ordering. One cannot a priori guess the properties of a such ordering, which would be radically different depending on the extremum computation method used (e.g. $\alpha$-trimmed lexicographical, cumulative distances, etc) as well as on the extremum employed (i. e. minimum or maximum). Besides, applying this procedure to the entire vector space would be extremely expensive in terms of computational complexity, but then again it needs to be done only once. Furthermore, one can also limit the procedure described in table 2 to the points of the multi-dimensional space occurring within the image to be processed, thus obtaining an "image-specific" ordering. Of course in this case the process needs to be repeated separately for every image.

\subsection{Setting $\alpha$}

As mentioned previously in section 3.1 , the choice of $\alpha$ directly controls the influence of each dimension on the computed extrema. Besides, finer control can be of course achieved by using different values of $\alpha$ for each dimension. In practice however, it is often necessary to set these arguments in an unsupervised fashion. Here we propose a simple parameter setting model based on the standard deviation $(\sigma)$ of each dimension.

Specifically, if the data are relatively concentrated with respect to their $i^{\text {th }}$ dimension, or in other words if this image channel does not contain much of the total variational information, we consider using a large $\alpha$ to be more pertinent, thus decreasing the influence of this dimension by carrying the majority of the input to the next dimension with minor trimming. Conversely, if the data are highly dispersed with respect to the other dimensions, meaning that this channel represents relatively important variational information, a small $\alpha$ would be used leading to major trimming. Given $n$ dimensions, one way of obtaining the corresponding $\alpha_{i}$ value of dimension $i$ would be:

$$
\forall i \in\{1, \ldots, n\}, \quad \alpha_{i}=1-\frac{\sigma_{i}}{\sum_{j=1}^{n} \sigma_{j}}
$$

where $\sigma_{j}$ denotes the standard deviation of dimension $j$.

\section{Application: Gaussian noise elimination}

In this section, we present the results of comparative tests carried out with the purpose of both illustrating the practical advantages of the proposed extrema computation scheme with respect to state-of-the-art methodologies, as well as asserting some of the remarks made in previous sections with experimental results.

The comparison of ordering approaches in multivariate mathematical morphology is relatively problematic since their performance depends on several criteria (e.g. data space, image data, employed operators, evaluation, etc). That is why here it was chosen to employ an easily quantifiable task, colour noise reduction, using four images with varying color distributions (figure 2). Moreover, since lexicographical ordering in general is most adequate for data 


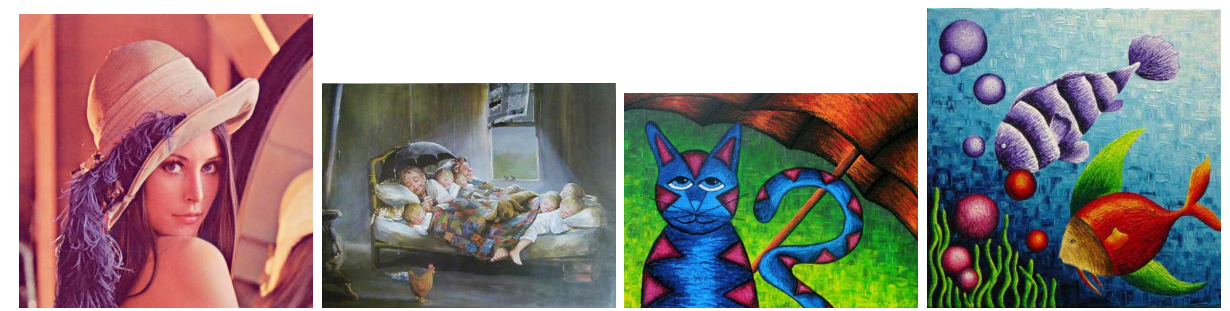

Figure 2. From left to right, Lenna (Lenna) $512 \times 512$, the image of happiness (Happy) $552 \times 421$ (by Abidin Dino), cat with umbrella (Cat) $576 \times 420$ (by Gamze Aktan) and the curious (Curious) $502 \times 512$ (by Gamze Aktan).

spaces where an inherent priority exists among the dimensions, using the RGB color space, where all three dimension are equally important, was considered inappropriate. Instead, an intuitive color space based on the notions of hue, saturation and luminance was employed, the improved HLS space based on the max-min norm [6], which remedies important drawbacks of the standard cylindrical HLS space (e. g. dependence between saturation and luminance, etc).

Since the human vision system is largely known to attribute greater importance to luminance variations with respect to chrominance, it was decided to set the lexicographical comparison order as $\mathrm{L}$ followed by $\mathrm{S}$ and finally $\mathrm{H}$ where all components are in $[0,1]$. As far as the periodicity of hue is concerned, its ordering was realized in terms of angular distances from a reference hue $h_{0}$ :

$$
h \div h_{0}=\left\{\begin{array}{cll}
\left|h-h_{0}\right| & \text { if } & \left|h-h_{0}\right|<0.5 \\
1-\left|h-h_{0}\right| & \text { if } & \left|h-h_{0}\right| \geq 0.5
\end{array}\right.
$$

which for the sake of simplicity was set as $h_{0}=0.0$. The hue values were then ordered according to their distances from $h_{0}$ :

$$
\forall h, h^{\prime} \in[0,1], \quad h<h^{\prime} \Leftrightarrow h^{\prime} \div h_{0}<h \div h_{0}
$$

where hues closer to $h_{0}$ are considered greater.

The images were corrupted in RGB with uncorrelated Gaussian noise ( $\sigma=0.125, \rho=0.0)$, and in order to quantify the relative performances, the relative normalized mean square error (RNMSE) was used, also in RGB:

$$
\operatorname{RNMSE}=\frac{\sum_{x, y}\left\|f(x, y)-f_{f}(x, y)\right\|^{2}}{\sum_{x, y}\left\|f(x, y)-f_{n}(x, y)\right\|^{2}}
$$

where $f(x, y), f_{n}(x, y), f_{f}(x, y)$ denote the vector pixels at position $(\mathrm{x}, \mathrm{y})$ respectively of the original, the noisy and filtered images, while $\|\cdot\|$ represents the Euclidean norm. The images were filtered using a SE of size $(3 \times 3)$ and the openclose close-open filter (OCCO):

$$
\operatorname{OCCO}(f)=\frac{1}{2} \phi(\gamma(f))+\frac{1}{2} \gamma(\phi(f))
$$

where $\phi$ and $\gamma$ denote respectively the closing and opening operators. The extrema computation approaches that were tested are luminance only (Lum), saturation only (Sat), hue only (Hue), lexicographical (Lex), $\alpha$-lexicographical with $\alpha=0.01$ ( $\alpha$-Lex), equation (4), $\alpha$-modulus lexicographical with $\alpha=10$ ( $\alpha$-modLex), equation (5), $\alpha$-trimmed lexicographical with $\alpha=0.45$ ( $\alpha$-trimmed-Lex) or computed according to equation (9) ( $\alpha$-trimmed-adaptive-Lex), and finally the total ordering resulting from the use of algorithm 2 with an $\alpha$-trimmed lexicographical minimum where $\alpha=0.45$ ( $\alpha$-trimmed-Lex-ordering). As to the fixed arguments, they were all set empirically as the values maximizing their performance based on the Lenna image. As a reference, the marginal ordering on RGB (MargRGB) was also included.

The filtering results are shown in table 3. Judging from the obtained values, the superiority of the marginal approach is obvious. By not being limited with the input vectors it is capable of approximating much better the original image, while the introduction of false colours is also unavoidable. Moreover, we can additionally observe the superiority of luminance over the other two dimensions, hence justifying our choice to set luminance at the first position of the lexicographical comparisons. Furthermore, one can also remark the priority attributed to the first dimension during lexicographical ordering, since its performance is very close to using only luminance. Of the first two $\alpha$ based approaches only $\alpha$-Lex leads to an improvement over Lum, with their difference however not being sufficiently large to draw sound conclusions.

The two trimming based extrema on the other hand, clearly outperform their counterparts. Despite resulting in pseudo-morphological operators, the set based extrema computation is presumed to aid their performance. Furthermore, although the empirically set constant $\alpha=0.45$ provides in average the best results, the adaptively set dimension specific arguments exhibit only slightly superior error levels. The image specific $\alpha$-trimmed lexicographical ordering however has been disappointing, clearly showing that further work is necessary on this option.

\section{Conclusion}

The extension of mathematical morphology to multivariate images is a challenging task, due to the ambiguous ordering relations of multidimensional data. Among 


\begin{tabular}{|c||c|c|c|c||c|}
\hline Method & Lenna & Happy & Cat & Curious & Average \\
\hline MargRGB & 14.07 & 14.57 & 18.44 & 24.38 & $\mathbf{1 7 . 8 7}$ \\
\hline Lum & 43.12 & 43.31 & 48.43 & 53.75 & 47.15 \\
Sat & 53.10 & 61.62 & 57.03 & 70.91 & 60.67 \\
Hue & 49.83 & 53.57 & 59.01 & 69.48 & 57.97 \\
\hline Lex & 43.20 & 43.31 & 48.50 & 53.72 & 47.18 \\
$\alpha$-Lex & 43.11 & 43.28 & 48.35 & 53.53 & 47.07 \\
$\alpha$-modLex & 43.51 & 43.92 & 48.74 & 53.79 & 47.49 \\
$\alpha$-trimmed-Lex & 29.97 & 39.74 & 35.77 & 45.16 & $\mathbf{3 7 . 6 6}$ \\
$\alpha$-trimmed-adaptive-Lex & 29.86 & 39.96 & 36.12 & 45.37 & 37.83 \\
$\alpha$-trimmed-Lex-ordering & 79.67 & 87.69 & 81.83 & 95.26 & 86.11 \\
\hline
\end{tabular}

Table 3. $100 \times R N M S E$ errors against uncorrelated Gaussian noise $(\sigma=0.125, \rho=0.0)$

the plethora of available ordering approaches, the lexicographical option has been widely experimented with in this regard, as it possesses desirable theoretical properties. In this paper we proposed an alternative solution to its main drawback, the highly asymmetric prioritization of data dimensions. The $\alpha$-trimming principle was used with this purpose, leading to more flexible inter-channel priority relations. Moreover, as with all set based extrema computation schemes lacking an underlying ordering relation, the proposed approach led to pseudo morphological operators, against which an iterative method was proposed in the end of ordering the data space.

The proposed approach was tested against state-of-theart ordering mechanisms used in color morphology, in the context of Gaussian noise reduction. Despite empirically set arguments for all compared methodologies, $\alpha$-trimming lexicographical extrema outperformed their counterparts, with the proposed adaptive $\alpha$ calculation model also proving to be a robust and unsupervised solution for parameter setting.

Future work will concentrate on the influence of $\alpha$ on applications other than noise reduction (e. g. classification of remote sensing imagery), as well as on more elaborate parametrization models. Moreover, given the relatively high error rates of the $\alpha$-trimmed lexicographical minimum based ordering, possible improvements to the preliminary version of algorithm 2 will be studied.

\section{References}

[1] J. Angulo. Unified morphological color processing framework in a lum/sat/hue representation. In C. Ronse, L. Najman, and E. Decencière, editors, Proceedings of the 7th ISMM, volume 30 of Computational Imaging and Vision, pages 387-396, Dordrecht, Germany, 2005. SpringerVerlag.

[2] E. Aptoula and S. Lefèvre. A comparative study on multivariate mathematical morphology. Pattern Recognition, 2007. (accepted for publication).

[3] V. Barnett. The ordering of multivariate data. Journal of the Statistical Society A, 139(3):318-355, 1976.
[4] J. Goutsias, H. J. A. M. Heijmans, and K. Sivakumar. Morphological operators for image sequences. Computer Vision and Image Understanding, 62(3):326-346, November 1995.

[5] A. Hanbury and J. Serra. Mathematical morphology in the CIELAB space. Image Analysis and Stereology, 21(3):201206, March 2002.

[6] A. Hanbury and J. Serra. Colour image analysis in 3d-polar coordinates. In International Conference on Image Processing and its Applications, Magdeburg, Germany, September 2003.

[7] G. Louverdis, M. Vardavoulia, I. Andreadis, and P. Tsalides. A new approach to morphological color image processing. Pattern Recognition, 35(8):1733-1741, September 2002.

[8] F. Ortiz, F. Torres, J. Angulo, and S. Puente. Comparative study of vectorial morphological operations in different color spaces. Proceedings of Intelligent robots and computer vision XX: Algorithms, Techniques, and Active Vision, 4572:259-268, November 2001.

[9] R. Öten and R. J. P. De Figueiredo. Adaptive alpha-trimmed mean filters under deviations from assumed noise model. IEEE Transactions on Image Processing, 13(5):627-639, May 2004.

[10] A. Plaza, P. Martinez, R. Perez, and J. Plaza. A new approach to mixed pixel classification of hyperspectral imagery based on extended morphological profiles. Pattern Recognition, 37(6):1097-1116, June 2004.

[11] C. Ronse. Why mathematical morphology needs complete lattices. Signal Processing, 21(2):129-154, October 1990.

[12] J. Serra. Anamorphoses and function lattices. In E. R. Dougherty, editor, Mathematical Morphology in Image Processing, chapter 13, pages 483-523. Marcel Dekker, New York, 1993. 